\title{
Rapidly-implementable optimizely-sizable fuzzy controller architectures: A performance analysis for semiconductor packaging two axes table
}

\author{
Zeyad A. Karam ${ }^{1}$, Shahad S. Ghintab ${ }^{2}$, Sami Hasan ${ }^{3}$ \\ ${ }^{1}$ Al-Nahrain University / College of Information Engineering / Department of Systems Engineering \\ ${ }^{2}$ AL-Mansour University / College of Engineering / Department of Communication Engineering \\ ${ }^{3}$ Al-Nahrain University / College of Information Engineering / Department of Systems Engineering
}

\section{Article Info}

Received Feb 6, 2019

\section{Keyword:}

$\mathrm{X}-\mathrm{Y}$ two axes table PI-PD controller

PID controller

Fuzzy type 2, MOALO

\begin{abstract}
The tendency of miniaturizing semiconductor products towards nano-size transistor in integrated chips has motivated this work on the semiconductor package. Consequently, Four Fuzzy PID controller architectures based on type 2 FLC are developed; the Interval Type-2 Fuzzy Logic PID, IT2FLC PID MOALO-based, IT2FLC PI-PD, and IT2FLC PI-PD MOALO controllers. These architectures are improved to overcome the inherent nonlinearity in $\mathrm{X}$ $\mathrm{Y}$ table models and capacitate the uncertainties of the parameters and the disturbances. Both controllers are designed to improve the desired position specification at minimum settling time (Ts), rise time (Tr), overshoot through minimization of oscillation and friction rejection during tracking the desired position trajectory. The ant lion optimization (ALO) algorithm has been efficiently solved optimization problems with minimum parameters and execution time. Hence, Multi-Objective Ant Lion Optimizer (MOALO) has been implemented to size the gains of the proposed controllers to get the desired position trajectory according to the required specification. A comparison with a related existing work shows minimal numerical values of improved transient specification response of $\mathrm{Tr}, \mathrm{Mp} \%$ and $\mathrm{Ts}$ for the MOALO- Based developed IT2 FLC PID and IT2 FLC PI-PD architectures. Observation of a higher Maximum Percentage of Enhancement settling time is noticed in both axes within the IT2FLC PI-PD architecture. Accordingly, transient performances of the four architectures have been significantly improved. The improvement is noticeable within the response of IT2FLC PI$\mathrm{PD}$ architecture. The Maximum Percentage of Enhancement in the $\mathrm{X}$-axis and Y-axis has been improved more than eight-fold and six-fold respectively using IT2FLC PI-PD architecture.
\end{abstract}

\section{Corresponding Author:}

Sami Hasan,

Systems Engineering Department, Al-Nahrain University, Iraq

Email: Sami.hasan@coie-nahrain.edu.iq

\section{Introduction:}

Currently, the XY table has been an important part of computer numeric controlled (CNC) machines of processing facilities [1] [2]. CNC is one of the bases that result in releasing the industrial in a fast way especially the last decades. One of these CNC architectures is the two-dimension (X-Y) motion table of semiconductor packaging. That consists of a two-degree-of-freedom (2DOF) motion. The XY table control system consists of an encoder sensor, controller and an actuator mechanism of a servo motor and a ball screw. 
$\mathrm{X}-\mathrm{Y}$ table has inherent Nonlinearities of friction and actuator power amplifier saturation. Consequently, The $\mathrm{X}-\mathrm{Y}$ table control system has to manage the imprecision positioning, inaccuracy tracking, sluggish transient response and steady-state error [3]. Particularly, conventional PID, time-optimal, sliding mode, adaptive, fuzzy logic, and neural network controllers have been introduced for the X-Y table motion control. The PID controller generally has low-performance compared to the above complicated high- performance design controllers [4].

The imprecision positioning and accurate tracking performance are mainly tackled by linear motor control in micro coordinate measuring machines, e.g., atomic force and scanning tunneling microscopes. The digital motor has become the typical actuator of the numerical control (NC) X-Y table for NC table robots [5].

The semiconductor industries, as wire bonding and die mounting, have great challenges of rapid trajectory tracking motions with highly accelerated responsiveness [6]. These challenges introduce stability and sensitivity issues.

The contributions of this paper are:

- $\quad$ Four implementable Fuzzy controller architectures are developed.

- $\quad \mathrm{X}-\mathrm{Y}$ table Nonlinearity and uncertainties are directly handled.

- The transient position specification has been optimized the desired position specification at minimum settling time (Ts), rise time (Tr), overshoot through minimization of oscillation.

- Minimization of oscillation and friction rejection

\section{Related existing works:}

Any X-Y positioning stage must have zero steady-state positioning errors using the advanced controller. In 2017, two Inspirational works have been reported, the first, Letong, et al., proposed a proportionalderivative (PD) controller combined with a disturbance observer that adopted for a designed magnet-driven nanopositioning stage table [7].

The second, Retas Z. et al., Proposed the fuzzy logic technique and cascade controller for improving the tracking performance of the XY table ball screw driving system [8].

In [9], Lin et al. presented "a Robust Recurrent-Neural-Network (RRNN) sliding-mode control for a biaxial motion mechanism to allow reference contour tracking. The biaxial motion mechanism is an XY table of a computer numerical control machine that is driven by two field-oriented control permanent-magnet synchronous motors".

In 2013, Giap and Kim presented a neural network control using an adaptive algorithm for an AC servo XY table. This algorithm is to compensate for the disturbances. Lyapunov theory improved system stability [10].

Patrick et al. presented the real-time controls of Non-Minimum Phase (NMP) XY Table system using trajectory Zero Phase Error Tracking Controller (ZPETC). Pietursewicz designed a hybrid predictive approach to robust control of milling machine XY table velocity was proposed with the results of simulation tests [11]. In [12], "the integral sliding mode controller is proposed to achieve output tracking of the time-varying reference signal for the $\mathrm{X}-\mathrm{Y}$ table and inertia wheel inverted pendulum". In [13], two field-control synchronous motors are actuated a CNC biaxial motion machine using adaptive recurrent-neural-network (ARNN) motion control system. A type-1 and the type-2 fuzzy set are characterized by a 2-D and 3-D membership function respectively [14].

Mirjalili et al. [15] investigated the ant lion optimization (ALO) algorithm. This algorithm has been efficiently solved optimization problems with minimum parameters and execution time. Multi-Objective Ant Lion Optimizer (MOALO) has been implemented to size the gains of the proposed controllers to get the desired position trajectory according to the required specification.

\section{X-Y table model:}

The X-Y table dynamic behavior may be accurately demonstrated through traditional theoretical modeling methods. Accordingly, dynamic modeling will be obtained using the bond graph method.

The voice coil motor VCMs in direct-drive XY stage may be dynamically modeled in the bond graph as shown in Fig. 1 and 2. 


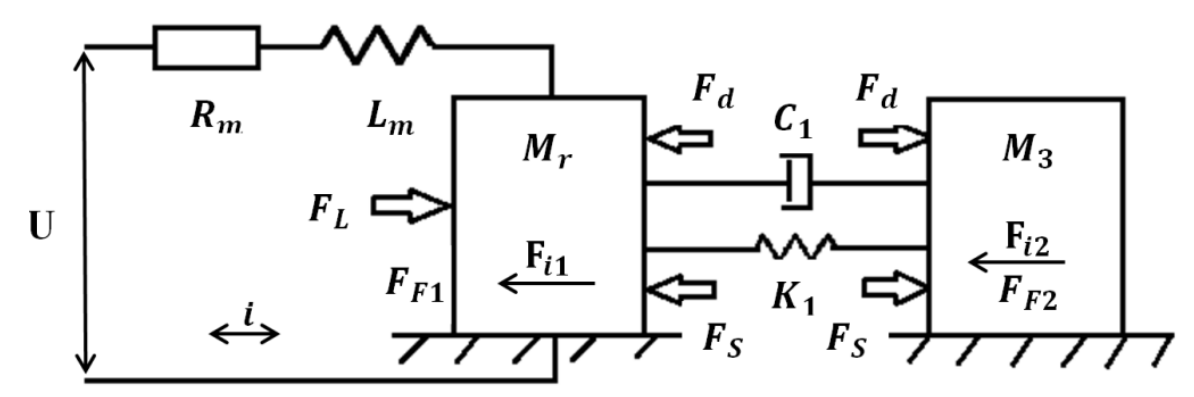

Fig. (1): The dynamic X axis model of the LVCMs XY stage of direct-drive

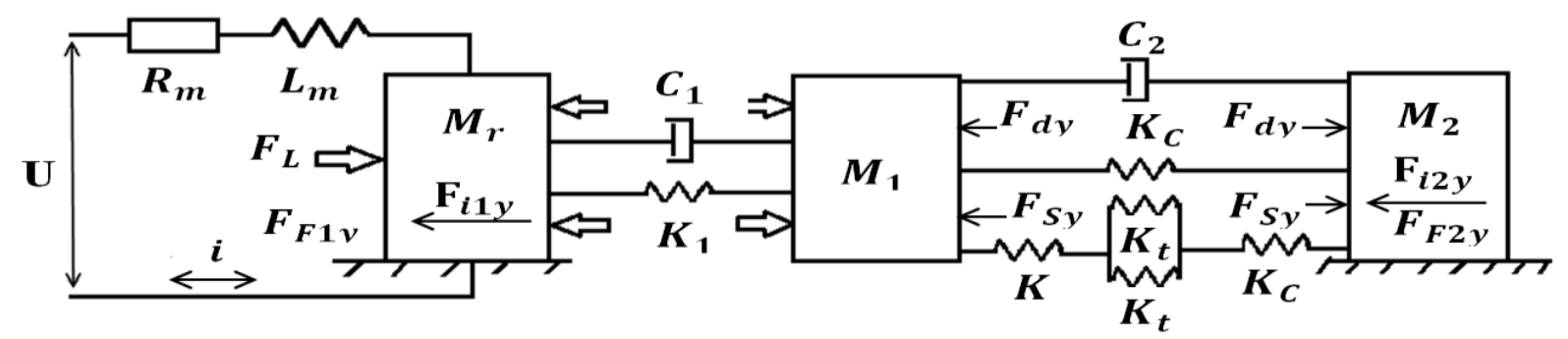

Fig. (2): The dynamic Y axis model of the LVCMs XY stage of direct-drive

Where $U$ : applied voltage, $M_{r}$ : coil mass, $M_{1}, M_{2}$ and $M_{3}: \mathrm{Y}$-axis fin, $\mathrm{Y}$-axis and $\mathrm{X}$-axis equivalent masses, $K_{1}$ : Stiffness, $C_{1}$ : damping, $\mathrm{K}$ : preload spring stiffness, $K_{t}$ : flexure hinge stiffness, $K_{c}$ : contact stiffness, $C_{2}$ $: \mathrm{Y}$-axis guiding fin and table equivalent damping, $F_{L}:$ coil output force, $F_{d}: \mathrm{X}$-axis damping force, $F_{S}: \mathrm{X}$-axis equivalent spring force, $F_{d y}:$ Y-axis damping force, $F_{s y}: \mathrm{Y}$-axis equivalent spring force, $F_{F 1}: \mathrm{X}$-axis coil friction, $F_{F 2}: \mathrm{X}$-axis table friction, $F_{F 1 y}: \mathrm{Y}$-axis coil friction, $F_{F 2 y}: \quad \mathrm{Y}$-axis table friction, $F_{i 1}: \mathrm{X}$-axis coil inertia force, $F_{i 2}: \mathrm{X}$-axis table inertia force, $F_{i 1 y}: \mathrm{Y}$-axis coil inertia force and $F_{i 2 y}: \mathrm{Y}-\quad$ axis inertia force.

The VCM consists of an internal resistor $R_{m}$ and the coil inductance, $L_{m}$ with the coil current $i$. The VCM bond graph may be mathematically modelled if the VCM motion velocity is $v$ and the corresponding generated force $F$ :

$U=R_{m} i+L_{m} \frac{d i}{d t}+K_{B} v$
$F=K_{F} i=M_{r} \frac{d v}{d t}+F_{L}$

Where $K_{B}:$ EMF coefficient and $K_{F}: F$ coefficient.

$M_{3} \frac{d^{2} x}{d t^{2}}+C_{1} \frac{d x}{d t}+K_{1} x+F_{L}-F_{f 2}$

$M_{y} \frac{d^{2} y}{d t^{2}}+C_{y} \frac{d y}{d t}+K_{y} y=F_{y}$

Where, $x$ : X-axis displacement, $M_{y}=\left[\begin{array}{cc}M_{1} & 0 \\ 0 & M_{2}\end{array}\right], C_{y}=\left[\begin{array}{cc}C_{1}+C_{2} & -C_{2} \\ -C_{2} & C_{2}\end{array}\right], K_{y}=\left[\begin{array}{cc}K_{1}+K_{y 2} & -K_{y 2} \\ -K_{y 2} & K_{y 2}\end{array}\right], y=$ $\left[\begin{array}{l}y_{1} \\ y_{2}\end{array}\right], F_{y}=\left[\begin{array}{c}F_{L} \\ -F_{F 2}\end{array}\right]$ and $K_{y 2}=\frac{2 K_{t} K_{r} K_{c}}{2 K_{t} K+2 K_{t} K_{c}+K K_{c}}+K_{c}$.

$y_{1}$ and $y_{2}:$ Y-axis displacements. Friction model is proposed as exponential:

$F_{F}(v f)=\operatorname{signum}\left(v_{f}\right) \cdot\left(F_{c}+\left(F_{s t}-F_{c}\right) \cdot e^{-\left|\frac{v_{f}}{v_{s}}\right|^{2}+\mu v_{f}}\right.$ 
Where $\mu$ : viscous friction coefficient, $v_{f} \& v_{s}$ : velocity and Stribeck velocity and $F_{c} \& F_{s t}$ : coulomb friction and static friction.

The state space model for the $\mathrm{X}$-axis may be expressed as:

$$
\begin{aligned}
& \dot{x}_{x}=A_{x} x_{x}+B_{x} u_{x} \\
& y_{x}=C_{x} x_{x}+D_{x} u_{x}
\end{aligned}
$$

$A_{x}=\left[\begin{array}{cccc}-\frac{R_{m}}{L_{M}} & -\frac{K_{B}}{M_{r}} & 0 & 0 \\ \frac{K_{B}}{L_{m}} & -\frac{C_{1}}{M_{r}} & -K_{1} & 0 \\ 0 & \frac{1}{M_{r}} & 0 & -\frac{1}{M_{3}} \\ 0 & \frac{C_{1}}{M_{r}} & 0 & -\frac{C_{1}+\mu}{M_{3}}\end{array}\right]$

$B_{x}=\left[\begin{array}{l}1 \\ 0 \\ 0 \\ 0\end{array}\right]$

$$
C_{x}=\left[\begin{array}{llll}
0 & 0 & 0 & \frac{1}{M_{3}}
\end{array}\right], D_{x}=0
$$

Y-axis state variables may express as [3]:

$$
\dot{x}_{y}=A_{y} x_{y}+B_{y} u_{y}
$$

$y_{y}=C_{y} x_{y}+D_{y} u_{y}$

Where,

$A_{y}=\left[\begin{array}{cccccc}-\frac{R_{m}}{L_{M}} & -\frac{K_{B}}{M_{r}} & 0 & 0 & 0 & 0 \\ \frac{K_{B}}{L_{m}} & -\frac{C_{1}}{M_{r}} & -K_{1} & \frac{C_{1}}{M_{1}} & 0 & 0 \\ 0 & \frac{1}{M_{r}} & 0 & -\frac{1}{M_{1}} & 0 & 0 \\ 0 & \frac{C_{1}}{M_{r}} & K_{1} & -\frac{C_{1}+C_{2}}{M_{1}} & -K_{e q} & \frac{C_{2}}{M_{2}} \\ 0 & 0 & 0 & \frac{1}{M_{1}} & 0 & -\frac{1}{M_{2}} \\ 0 & 0 & 0 & \frac{C_{2}}{M_{1}} & K_{e q} & -\frac{C_{2}+\mu}{M_{1}}\end{array}\right]$

$B_{y}=\left[\begin{array}{l}1 \\ 0 \\ 0 \\ 0 \\ 0 \\ 0\end{array}\right], C_{x}=\left[\begin{array}{llllll}0 & 0 & 0 & 0 & 0 & \frac{1}{M_{2}}\end{array}\right], D_{y}=0$.

Where $K_{e q}=K_{C}+\frac{2 K_{t} K K_{C}}{2 K_{t}+K+K_{C}}$.

The above parameters of the small scale system may have the numerical values of Table 1 . 
Table.1. X-Y table system parameters [1]

\begin{tabular}{|c|c|}
\hline System parameters & Values and units \\
\hline$R_{m}$ & $16 \Omega$ \\
\hline$L_{m}$ & $0.027 \mathrm{H}$ \\
\hline$K_{f}$ & $86 \mathrm{~N} / \mathrm{A}$ \\
\hline$K_{B}$ & $116.6 \mathrm{~N} / \mathrm{A}$ \\
\hline$M_{1}$ & $1.205 \mathrm{~kg}$ \\
\hline$M_{2}$ & $1.2 \mathrm{~kg}$ \\
\hline$M_{3}$ & $4.006 \mathrm{~kg}$ \\
\hline$C_{1}$ & $110 \mathrm{~N} . \mathrm{s} / \mathrm{m}$ \\
\hline$C_{2}$ & $110 \mathrm{~N} . \mathrm{s} / \mathrm{m}$ \\
\hline$K_{1}$ & $2.6 * 10^{\wedge} 7 \mathrm{~N} / \mathrm{m}$ \\
\hline$K_{e q}$ & $1.2 * 10^{\wedge} 5 \mathrm{~N} / \mathrm{m}$ \\
\hline$F_{c}$ & $11 \mathrm{~N}$ \\
\hline$F_{s t}$ & $9 \mathrm{~N}$ \\
\hline$v_{s}$ & $0.04 \mathrm{~m} / \mathrm{s}$ \\
\hline$\mu$ & $120 \mathrm{~N} . \mathrm{s} / \mathrm{m}$ \\
\hline
\end{tabular}

\section{Type-2 fuzzy sets and IT2FLC Systems:}

A type-2 fuzzy set is specified by a 3-D MF that includes a footprint of uncertainty (FOU). Hence, type-2 fuzzy sets have provided additional degrees of freedom for a superior uncertainty model. Interval type-2 FLCs may handle high uncertainty level applications of mobile robot control [16]. The inherent uncertainties may be handled using IT2FLC System (IT2FLS) [17]. A typical type-2 fuzzy membership function is consist of two type-1 fuzzy membership functions as shown in Fig.2. [18].

The uncertainty in the primary membership of a type-2 fuzzy set can be defined as a bounded region so-called Footprint of Uncertainty (FOU) between these two type-1 fuzzy membership functions, one is an "upper membership function" (UMF) and the other is "lower membership functions" (LMF), mathematically FOU can be described as the union region between LMF and UMF, footprint can be described as $\mathrm{X}$

$\operatorname{FOU}(\tilde{X})=\bigcup_{X \in D_{X}} J_{X}$

Where, $(\tilde{X})$ is an interval type- 2 fuzzy when all $\mu_{\tilde{X}}(x, u)=1$. As described in type-the 1 fuzzy logic system, a type-2 fuzzy contains also a fuzzifier block, rule-base, inference engine and substitute defuzzifier at the output processor. This last includes furthermore a type-reducer, and it generates a regular type-1 output [18].

The IT2FIS diagram as shown in Fig.3 [14] may consist of;

1) Fuzzifier

The vector of crisps inputs $x^{T}=\left(x_{1} \ldots x_{p}\right)^{T}$ is fuzzified firstly under the fuzzifier block where it is mapped into type-2 fuzzy sets $\tilde{X}$

2) Rules

As indicated for the type-1 fuzzy, a Type-2 Fuzzy System has also IF-THEN rule architecture however the consequent in fuzzy type-2 is described as follows [20]:

$R^{n}:$ if $\left(x_{1}\right)$ is $\left(\tilde{X}_{1}^{n}\right)$ and ..... and $\left(x_{p}\right)$ is $\left(\tilde{X}_{p}^{n}\right)$ then $(y)$ is $\left(Y^{n}\right) \quad n=0,1, \ldots \ldots L L \in N$ 
Where $\tilde{X}_{p}^{n}(i=1, \ldots, P)$ are interval type-2 fuzzy system and $Y n$ is the interval output. For an input vector $x=\left(x_{1}, x_{2}, \ldots x_{p}\right)$ of the $p^{\text {th }}$ inputs, $n$ is the number of rules.

3) Inference

In the type-2 fuzzy system, the inference engine gives a mapping from the fuzzified input type-2 fuzzy sets to the defuzzification block after combining with the rules.

$F^{l}\left(X^{\prime}\right)=\left[\underline{f}^{l}\left(X^{\prime}\right), \bar{f}^{l}\left(X^{\prime}\right)\right]=\left[\underline{f}^{l}, \bar{f}^{l}\right]$

where

$\underline{f}^{l}\left(X^{\prime}\right)=\underline{\mu_{F_{1}^{1}}^{\prime}}\left(x_{1}^{\prime}\right) * \ldots \ldots * \underline{\mu_{F_{p}^{1}}^{\prime}}\left(x_{p}^{\prime}\right)$

and

$\bar{f}^{l}\left(X^{\prime}\right)=\overline{\mu_{F_{1}^{1}}^{\prime}}\left(x_{1}^{\prime}\right) * \ldots \ldots * \overline{\mu_{F_{p}^{1}}^{\prime}}\left(x_{p}^{\prime}\right)$

4) Type-reducer

The function for the center of sets, called $Y_{\cos }$ is expressed as [18]:

$Y_{\text {cos }}(X)=\left[y_{1}, y_{r}\right]$

$\left[y_{1}, y_{r}\right]=\int y^{1} \in\left[y_{l}^{1}, y_{r}^{1}\right] \ldots \int y^{1} \in\left[y_{l}^{M}, M\right]$

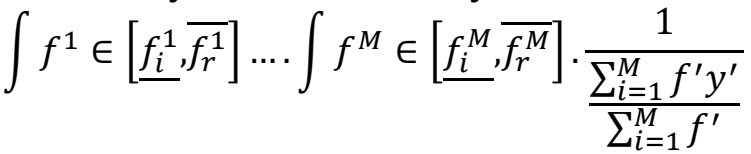

And consequent set can be described as:

$C_{\tilde{G}^{i}}=\int \theta_{1} \in J_{y_{1}} \ldots \int \theta_{N} \in J_{y_{N}} \frac{1}{\frac{\sum_{i=1}^{N} y_{i} \theta_{i}}{\sum_{i=1}^{N} \theta_{i}}}=\left[y_{l}^{i}, y_{r}^{i}\right]$

Eq. (22) should be evaluated before the calculation of $Y_{\cos }(x)$.

$y_{l}^{i}=\frac{\sum_{i=1}^{M} f_{l}^{i} y_{l}^{i}}{\sum_{i=1}^{M} f_{l}^{i}} \quad$ and $\quad y_{r}^{i}=\frac{\sum_{i=1}^{M} f_{r}^{i} y_{r}^{i}}{\sum_{i=1}^{M} f_{r}^{i}}$

A Karnik and Mendel type reducer is used for the type reduction algorithm. It should be noted that, although other type reducer algorithms are tested, Karnik and Mendel's algorithm shows satisfactory performance.

5) Defuzzifier

An interval set, which is called $Y_{\text {cos }}$, can be obtained from the type-reducer. In order to defuzzify this set an average of $y_{l}$ and $y_{r}$ is used, by the way defuzzifier output is given as:

$y(X)=\left(y_{l}+y_{r}\right) / r$

\section{The architectures of the IT2FLC PID Controller with a cascade connection of IT2FLC PI-PD controllers:}

Traditional PID and PI-PD Controllers may not efficiently be performed for systems of nonlinear, higherorder-time-delayed linear and vague no precise mathematical models. Consequently, a class of non-traditional fuzzy type 2 PI-PD controllers has been reported [19]. The control input variables may be fuzzified, then, inputted to the control algorithm of the FLC. Since the X-Y table has high nonlinearity, FLC type 2 has the ability to deal with this nonlinearity and to achieve tracking performance such as minimum overshoot, 
minimum oscillation, and friction rejection. An FLC of PI-PD type 2 is designed as shown in Fig.3. The inputs of the controller are the (error (e) and the rate of error (è)).

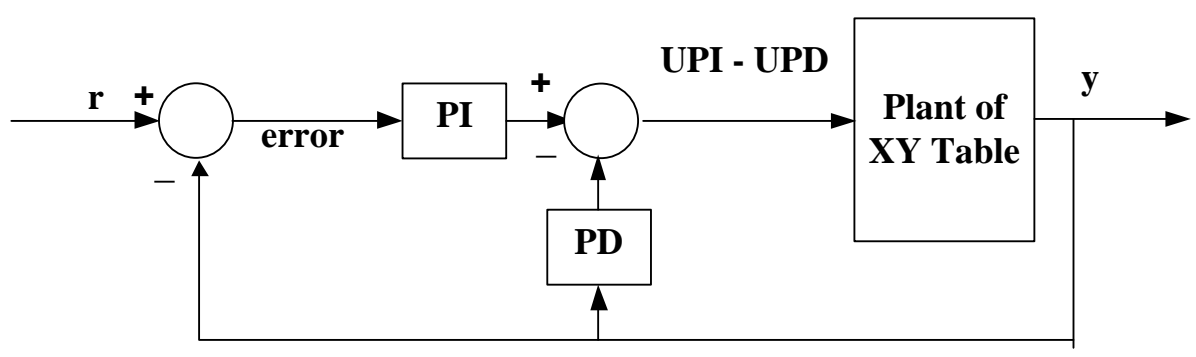

Fig. (3): the conventional continuous-time PI-PD control system [PI-PD CONTROLLER].

The developed software is called Type-2 Fuzzy Logic Toolbox is used a collection of MATLAB. In the type-2 fuzzy rule two inputs were chosen by representing the error and the error variation under the linguistic representations as rule base; The linguistic variables of the membership functions of the FLC are; Negative Big (NB), Negative Medium (NM), Negative Small (NS), Zero (Z), Positive Small (PS), Positive Medium (PM), and Positive Big (PB) as summarized in Table.2. That selected by several trials to reach the best response. The FOU is chosen between 1 and 0.5 for all the membership functions. Seven Gaussian shaped membership functions are used for inputs and output, see Fig.4. Then fuzzy inference engine infers the input variables to a suitable fuzzy set. And, an output signal is obtained by defuzzification. Takagi-Sugeno (TS), is chosen as the method of fuzzy inference with an output range of $-1 /+1$ for the negative and positive respectively and $-0.8 / 0.8$ for the negative medium and positive medium respectively as shown in Fig.5. The inputs and output scaling factors are defined as proportional gain (KP), derivative gain (KD), After-derivation gain (KA), integration gain (KI), After-integral gain (KS) and output gain (KF)). The universe of discourse for (e, $\dot{e}$, and the output) is taken within $(-10,10)$. Also, the values of the gains (KP KD KA KI KS KF) are sized by using MOALO to achieve the desired specifications such as minimum settling time (Ts), minimum rise time (Tr), minimum overshoot through the minimization of oscillation and friction rejection.

Table 2: The rule table of FLC.

\begin{tabular}{|c|c|c|c|c|c|c|c|}
\hline $\begin{array}{c}\dot{e} \\
\boldsymbol{e}\end{array}$ & NB & NM & NS & Z & PS & PM & PB \\
\hline NB & NB & NB & NB & NB & NM & NS & Z \\
\hline NM & NB & NB & NB & NM & NS & Z & PS \\
\hline NS & NB & NB & NM & NS & Z & PS & PM \\
\hline Z & NB & NM & NS & Z & PS & PM & PB \\
\hline PS & NM & NS & Z & PS & PM & PB & PB \\
\hline PM & NS & Z & PS & PM & PB & PB & PB \\
\hline PB & Z & PS & PM & PB & PB & PB & PB \\
\hline
\end{tabular}




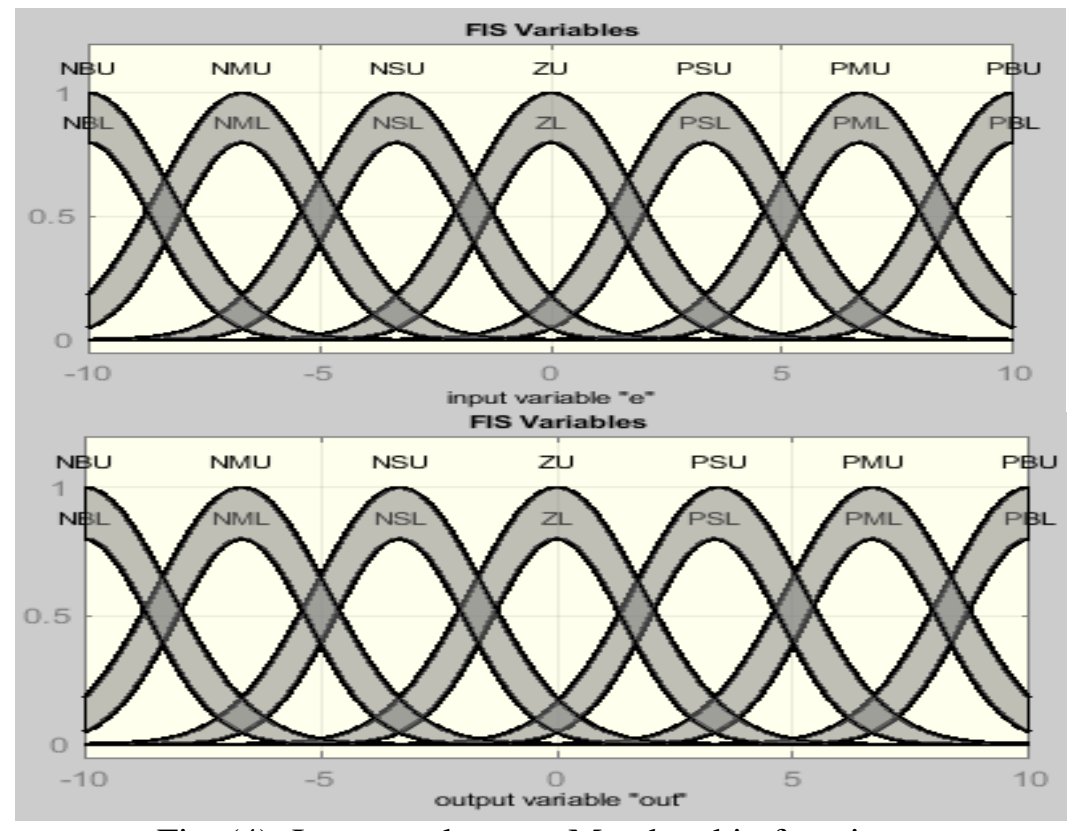

Fig. (4): Inputs and output Membership functions.

The Simulink of the control block diagram Interval Type-2 PID FLC for the X and Y-axes servomechanisms showed in fig.5and fig.6. Respectively.

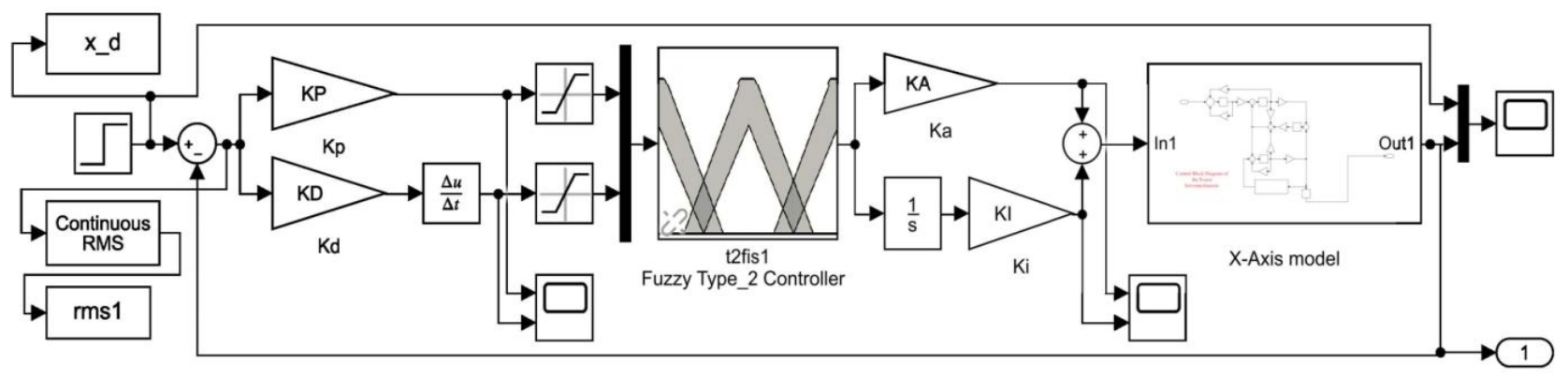

Fig. (5): Interval Type-2 PID Fuzzy Logic Controller of the X-axis Model.

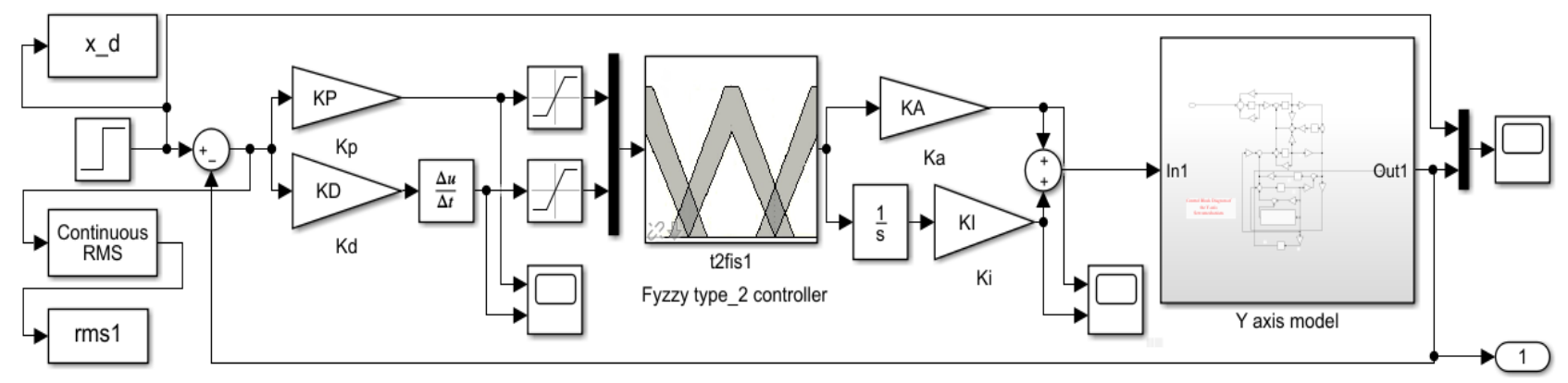

Fig (6): Interval Type-2 PID Fuzzy Logic Controller of the Y-axis Model.

The second controller used in this work is a fuzzy type 2 PI-PD position controller. In comparison with traditional PID, This controller provides higher damping and stiffness [20]. The cascade control scheme, as shown in fig.7, is an integrated structural design using a PID controller for the purpose of accurate, desired control action in the presence of load change and variable process dynamics. Cascade control system is designed easily and provides a large performance improvement over a single control system. If a secondary measurable variable is available then it is advantageous to use cascade control in comparison to the single element control system. Cascade control system consists of two control loops; an inner loop or secondary and 
outer loop or primary loop and two controllers rather than a single PID controller. Inner loop controller is called secondary or slave and primary loop controller are called primary or master controller [21].

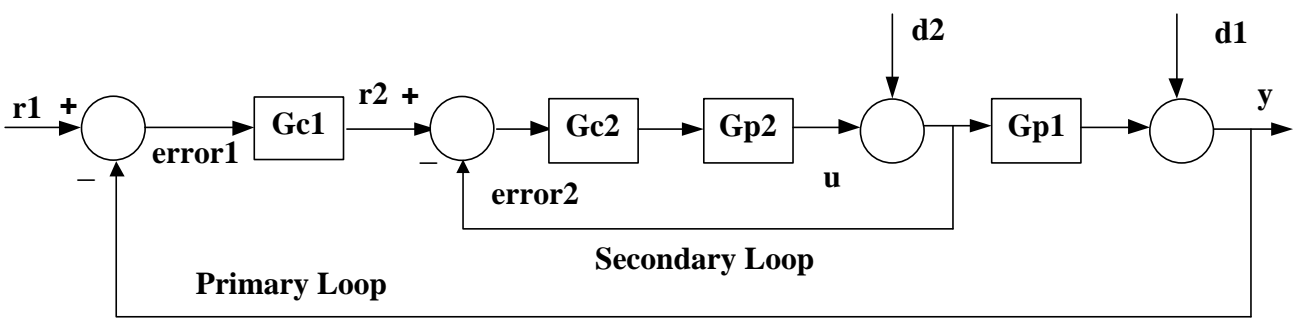

Fig. (7): Cascade control system [21]

However, Fig.8. and 9 Shows the block diagram for the closed loop dynamic model controlled by a cascade connection of fuzzy type 2 PI and PD position controllers for $\mathrm{X}$ and $\mathrm{Y}$ axis. The parameters of the cascade controllers are sized by MOALO.

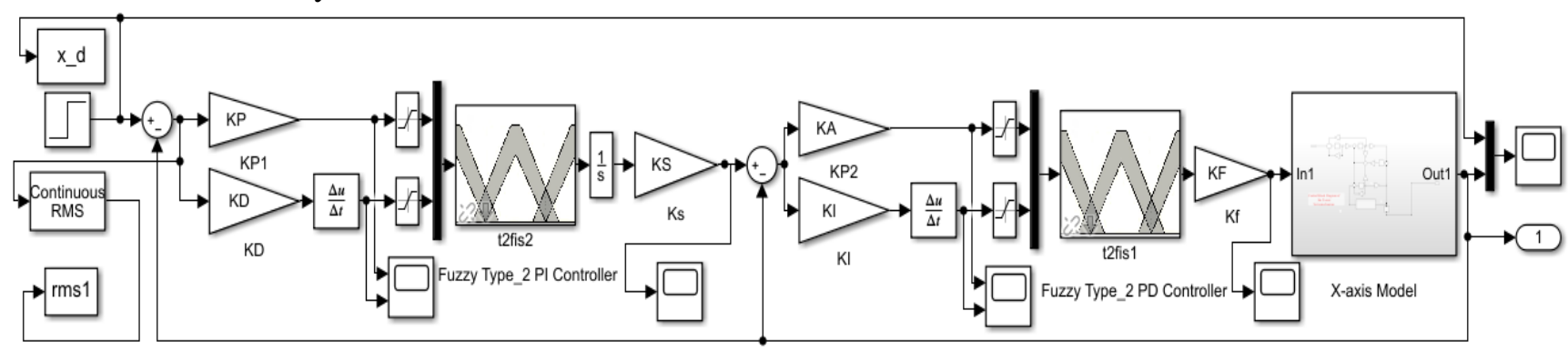

Fig. (8): Control block diagram of the $\mathrm{X}$-axis servomechanism.

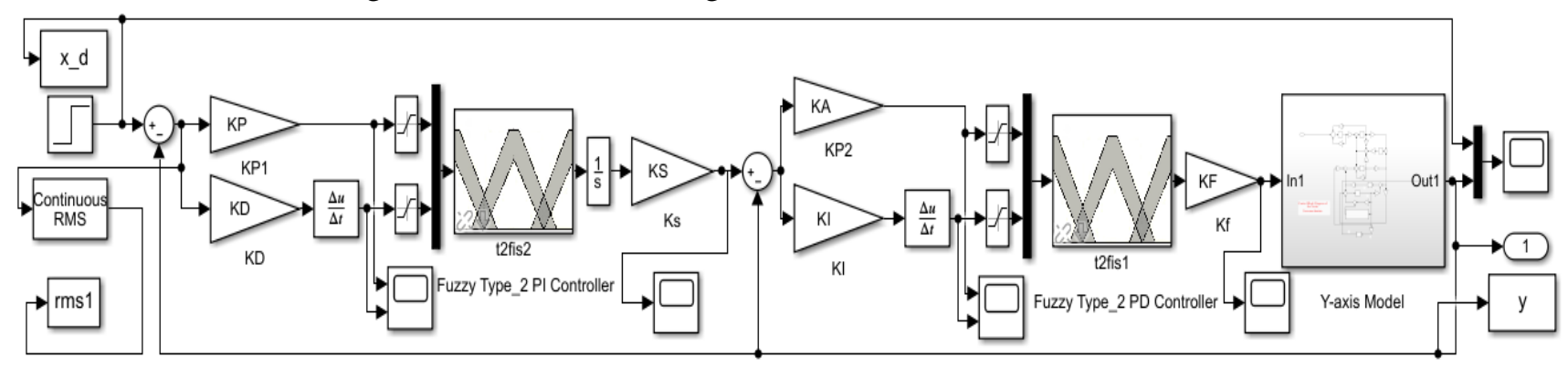

Fig. (9): Control block diagram of the Y-axis servomechanism.

The MOALO used as (off-line) optimization algorithm to find the best gains of the Interval Type-2 PID Fuzzy Logic Controller and the second one is cascade connection of IT2 FLC PI and PD position controllers, in order to reach accurate responses via minimizing the overshoot in position responses with minimized settling time and rise time. The MOALO runs off-line, thus computing time is not as important as in real-time control. All elements of MOALO are changed for many tests until reach the largest enhancement at minimum fitness function. Fig.10. shows the block diagram for MOALO-based controller.

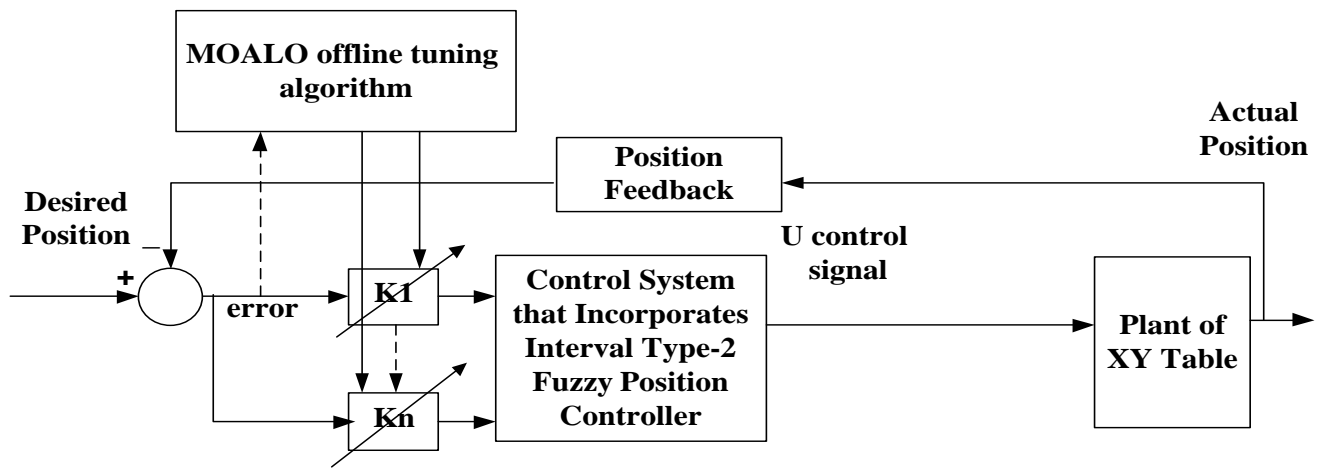

Fig. (10): Interval Type-2 Fuzzy Position Controller architecture MOALO based block diagram [22]. 


\section{Simulation Results and Analysis:}

The outstanding performance of our proposed fuzzy type 2 controllers is demonstrated by computer simulations on an X-Y table system. It is difficult to enhance the position tracking performance using the controller sized manually. The process of tuning the controllers gain consumes time and efforts. In this research, MOALO algorithm used to size the gains of the controllers in order to reach the best position performance in offline method. To get more accurate position responses, the values of the gains KP, KD, KA, and $\mathrm{KI}$ for the first controller and KP, KD, KA, KI, KS, and KF for the second controller are sized by MOALO to achieve the desired specifications based on the mentioned fitness. There are more than ten tests of tuning for controllers gain to get the desired position trajectory in the minimized control signal speciation. The algorithm stops iterating either when an ant found a solution or when a maximum number of iterations have been performed.

The optimal position tracking responses in the $\mathrm{x}, \mathrm{y}$-axis are shown in Fig.13. and Fig.14 respectively using the IT2FLC PID Controller based on MOALO algorithm that utilized the finesses of Fig. 11 and 12 respectively.

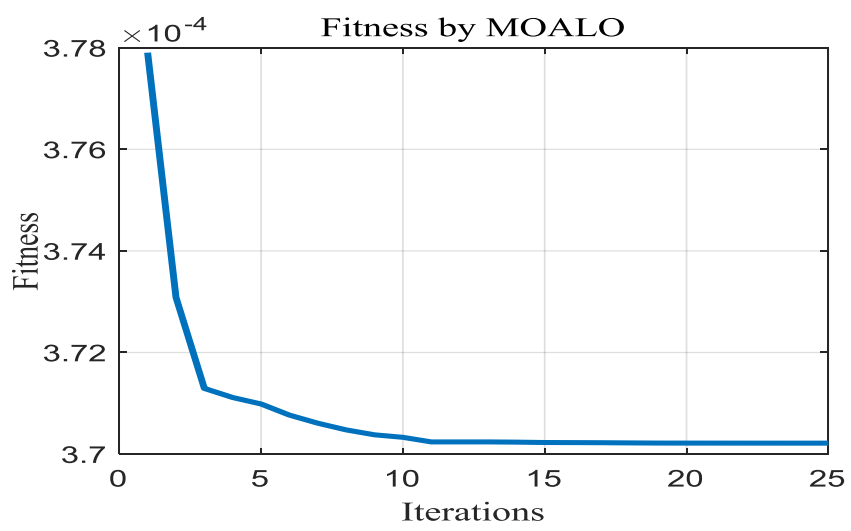

Fig. (11): the fitness index obtained by MOALO with Interval Type-2 PID-FLC for X-axis.

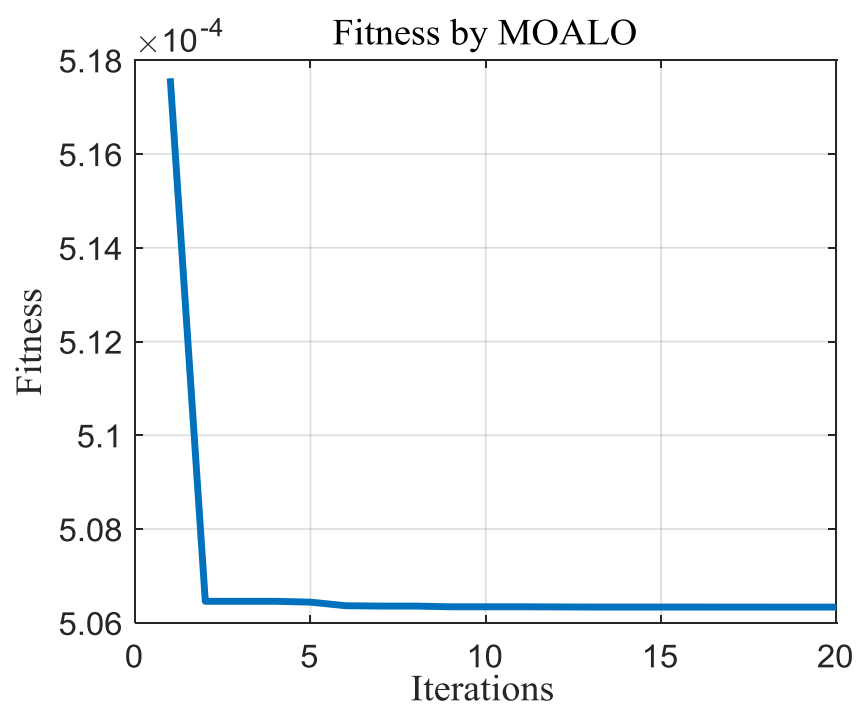

Fig. (12): the fitness index obtained by MOALO with Interval Type-2 PID-FLC for Y-axis. 


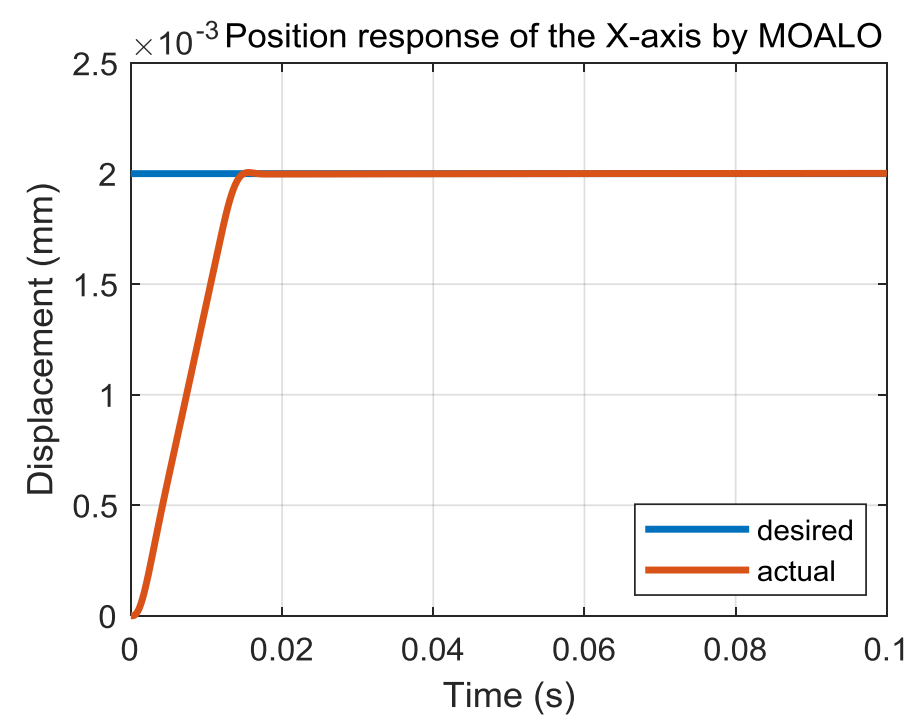

Fig. (13): Position response of the $\mathrm{X}$-axis motion with Interval Type-2 PID-FLC.

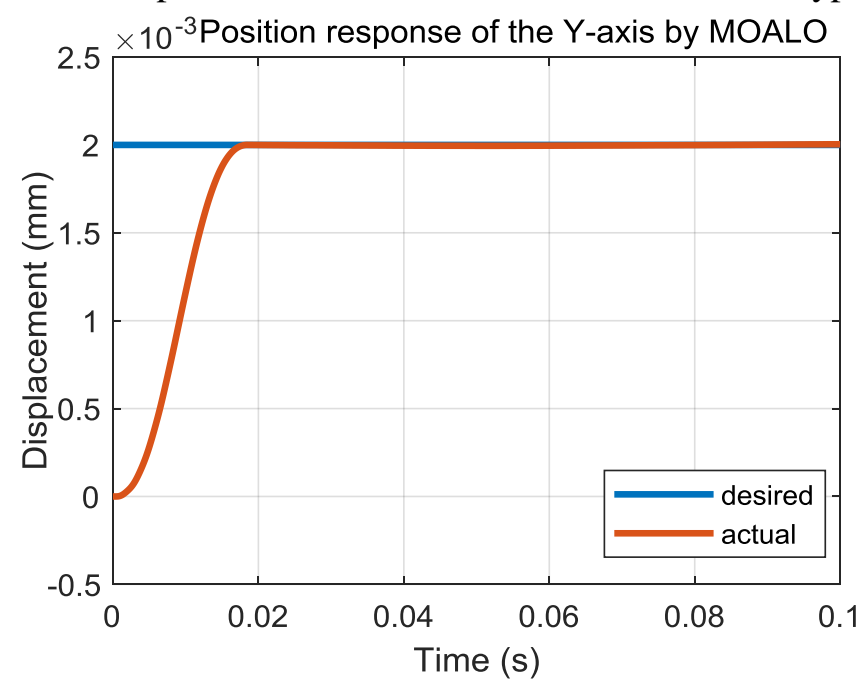

Fig. (14): Position response of the Y-axis motion with Interval Type-2 PID-FLC.

The same scenario is applied to PI-PD controller, where the optimal position tracking responses in the $\mathrm{x}, \mathrm{y}$ axis are shown in Fig.17. and Fig.18 respectively using the IT2FLC PI-PD Controller based on MOALO algorithm that utilized the finesses of Fig.15 and 16 respectively.

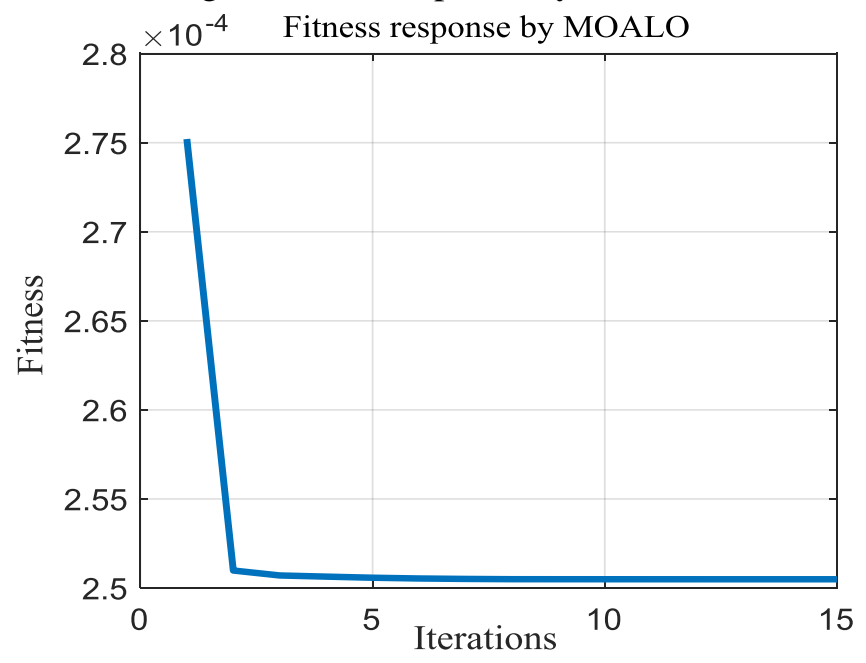

Fig. (15): The fitness index obtained by MOALO with Interval Type-2 cascade PI-PD- MOALO based.-FLC for X-axis. 


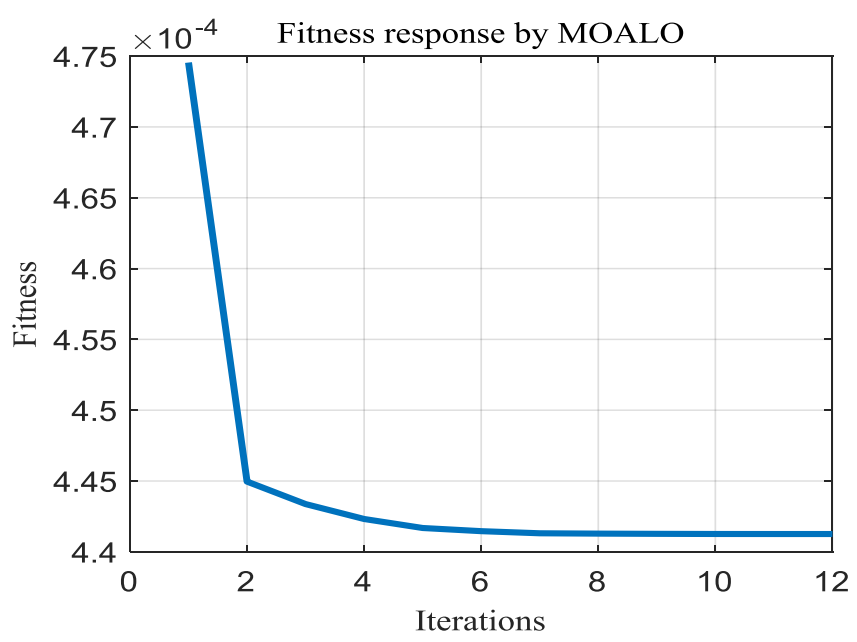

Fig. (16): The fitness index obtained by MOALO with Interval Type-2 cascade PI-PD- MOALO based.-FLC for Y-axis.

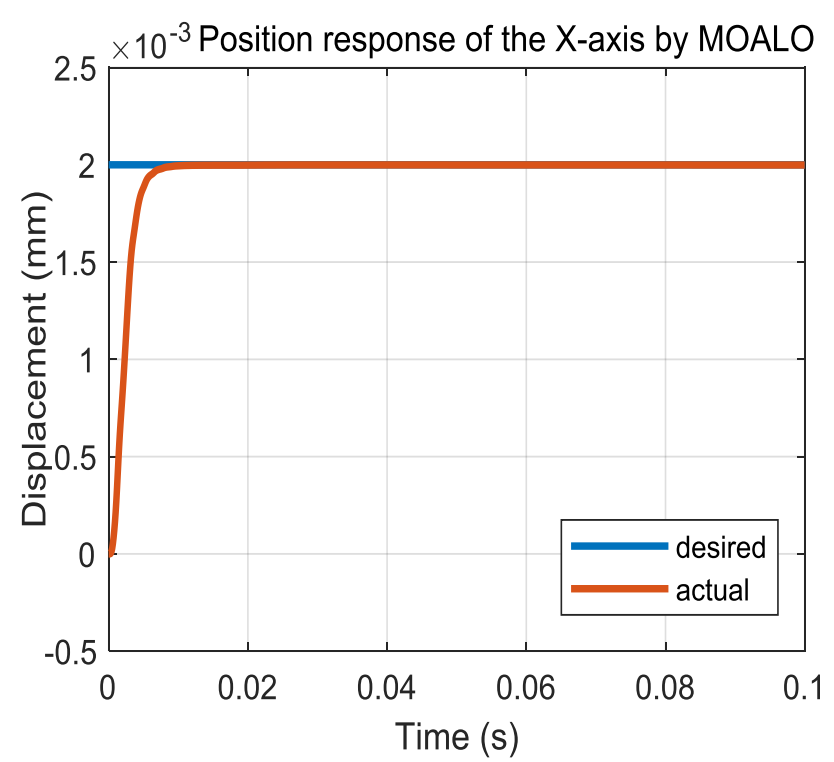

Fig. (17): Position response of the X-axis motion with IT2 FLC cascade PI-PD- MOALO based.

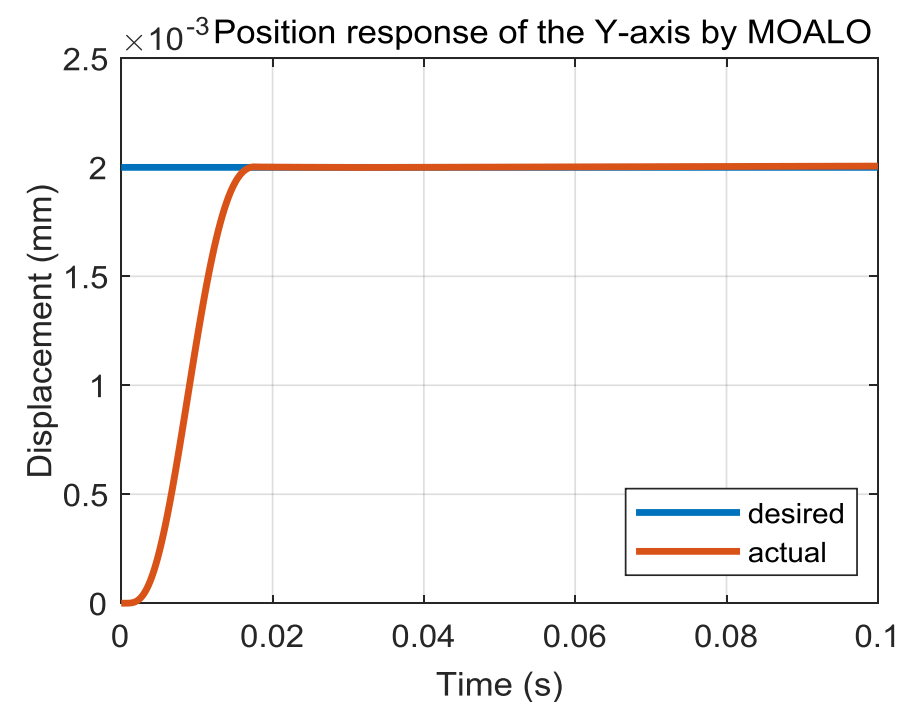

Fig. (18): Position response of the Y-axis motion with IT2 FLC cascade PI-PD- MOALO based. 
Table. 3 summarizes the MOALO-sized controllers for each axis. Where the number of agents and the number of iterations is selected so that the MOALO algorithm sized the global fitness. Consequently, the Percentage of Enhancement of IT2FLC PI-PD is super performed that of IT2FLC PID according to the architectural modification.

Table.3. Optimized Fitness for the four IT2 FLC Architectures.

\begin{tabular}{|c|c|c|c|c|c|}
\hline $\begin{array}{c}\text { MOALO Based } \\
\text { Controller Type }\end{array}$ & $\begin{array}{c}\text { Fitness by } \\
\text { Interval } \\
\text { Type-2 } \\
\text { Fuzzy PID }\end{array}$ & $\begin{array}{c}\text { Best Global } \\
\text { fitness by } \\
\text { MOALO }\end{array}$ & $\begin{array}{c}\text { Number of } \\
\text { Iterations }\end{array}$ & $\begin{array}{c}\text { Number } \\
\text { of Agents }\end{array}$ & $\begin{array}{c}\text { Percentage of } \\
\text { Enhancement }\end{array}$ \\
\hline \multicolumn{7}{|c|}{ X-axis Motion Control } \\
\hline IT2FLC PID & 0.0005296 & 0.0003702 & 25 & 35 & $30.098 \%$ \\
\hline IT2FLC PI-PD & 0.0005296 & 0.0002504 & 15 & 80 & $52.719 \%$ \\
\hline \multicolumn{7}{|c|}{ Y-axis Motion Control } \\
\hline IT2FLC PID & 0.0005296 & 0.0005060 & 20 & 70 & $4.456 \%$ \\
\hline IT2FLC PI-PD & 0.0005296 & 0.0004336 & 12 & 100 & $18.126 \%$ \\
\hline
\end{tabular}

Table. 4 illustrate the optimized parameters KP, KD, KA, and KI for PID controller and KP, KD, KA, KI. KS, and KF for PI-PD controller. These parameters have been obtained using MOALO algorithm to follow the desired position trajectory.

Table.4 Gains obtained by MOALO method for the Interval Type-2 Fuzzy controller Architectures.

\begin{tabular}{|c|c|c|c|c|c|c|}
\hline \multicolumn{7}{|c|}{$\begin{array}{c}\text { X-axis Motion MOALO Based Control } \\
\text { IT2 FLC PID }\end{array}$} \\
\hline Gain & $K_{P}$ & \multicolumn{2}{|c|}{$K_{d}$} & $K$ & \multicolumn{2}{|c|}{$K_{I}$} \\
\hline Value & 26914 & \multicolumn{2}{|c|}{36} & 72 & \multicolumn{2}{|r|}{93} \\
\hline \multicolumn{7}{|c|}{ IT2 FLC PI-PD } \\
\hline Gain & $K_{P l}$ & $K_{d}$ & $K_{P 2}$ & $K_{I}$ & $K_{S}$ & $K_{F}$ \\
\hline Value & 12000 & 0.1 & 273 & 79 & 0.7 & 200 \\
\hline \multicolumn{7}{|c|}{ Y-axis Motion Control } \\
\hline \multicolumn{7}{|c|}{ IT2 FLC PID } \\
\hline Gain & $K_{P}$ & \multicolumn{2}{|c|}{$K_{d}$} & \multicolumn{2}{|c|}{$K_{A}$} & $K_{I}$ \\
\hline Value & 6640 & \multicolumn{2}{|c|}{0.1} & 20 & \multicolumn{2}{|c|}{199.1} \\
\hline \multicolumn{7}{|c|}{ IT2 FLC PI-PD } \\
\hline Gain & $K_{P 1}$ & $K_{d}$ & $K_{P 2}$ & $K_{I}$ & $K_{S}$ & $K_{F}$ \\
\hline Value & 3626.2 & 1.1 & 19 & 9 & 1.26 & 70 \\
\hline
\end{tabular}

A comparison with a related existing work [1] is summarized in Table 5 and depicted in Fig. 18 and 19. In TABLE 5, the transient specification of Tr, Mp\% and Ts for the MOALO- Based developed IT2 FLC PID and IT2 FLC PI-PD architectures show lower numerical values of improved transient response. Observation of a higher Maximum Percentage of Enhancement settling time is noticed in both axes within the IT2FLC PI-PD architecture. 
Table. 5 Optimal values for each criterion to the proposed and previous works with enhancement.

\begin{tabular}{|c|c|c|c|c|}
\hline \multirow{2}{*}{$\begin{array}{c}\text { Control } \\
\text { Specification }\end{array}$} & $\begin{array}{c}\text { IT2 FLC PID- } \\
\text { MOALO Based }\end{array}$ & $\begin{array}{c}\text { IT2 FLC PI-PD - } \\
\text { MOALO Based }\end{array}$ & Previous work[1] & $\begin{array}{c}\text { Maximum } \\
\text { Percentage of } \\
\text { Enhancement }\end{array}$ \\
\hline \multicolumn{5}{|c|}{ X-axis Motion Control } \\
\hline$T_{r}(\mathrm{~ms})$ & 12.3 & 4.3 & - & $65.040 \%$ \\
\hline$M_{p} \%$ & 0.09 & 0 & 7 & Cancel the $M_{p}$ \\
\hline$T_{S}(\mathrm{~ms})$, Criteria 2\%. & 13.3 & 6.5 & 64 & $89.843 \%$ \\
\hline \multicolumn{5}{|c|}{ Y-axis Motion Control } \\
\hline$T_{r}(\mathrm{~ms})$ & 14.1 & 12.6 & - & $10.638 \%$ \\
\hline$M_{p} \%$ & 0 & 0.01 & 8 & Cancel the $M_{p}$ \\
\hline$T_{S}(\mathrm{~ms})$, Criteria 2\%. & 16.2 & 14.5 & 62 & $76.612 \%$ \\
\hline
\end{tabular}
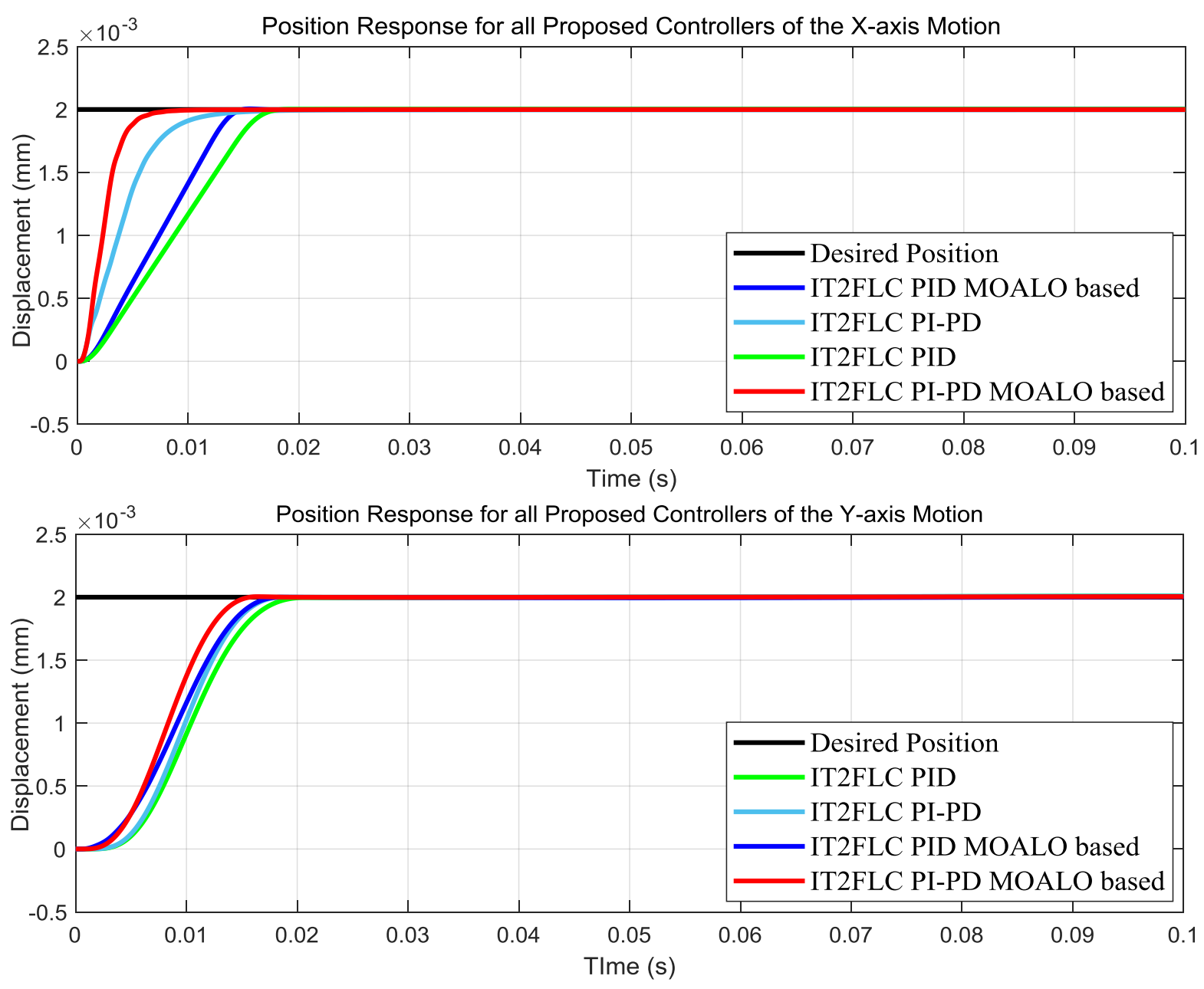

Fig. (19): the position response of the $\mathrm{X}$ and $\mathrm{Y}$ axes by all proposed controllers.

Accordingly, transient performance of the four architectures has been significantly improved in decreasing overshoot percentage, raising and settling times. The improvement is noticeable within the response of IT2FLC PI-PD architecture. The Maximum Percentage of Enhancement in the X-axis has been improved more than eight-fold using IT2FLC PI-PD architecture compared to [1].

\section{Conclusion}

Four fuzzy PID controller architectures have been developed, implemented and optimized to produce; IT2FLC PID, IT2FLC PID MOALO-based, IT2FLC PI-PD, and IT2FLC PI-PD MOALO. These four fuzzy PID architectures have been improved the transient and steady-state error responses. Accordingly, IT2FLC PIPD architecture has a superior transient response of more than eight-fold. 
The future work is to implement the developed fuzzy controllers in an FPGA hardware [24], [25], [26], [27] and [28] as reconfigurable architectures.

\section{References:}

[1] Wang, Fujun, et al. "Dynamic modeling and control of a novel XY positioning stage for semiconductor packaging." Transactions of the Institute of Measurement and Control 37.2 (2015): 177-189.

[2] Victor Vladareanu, et al., "Adaptive neural network fuzzy inference system for HFC processes." Periodicals of Engineering and Natural Sciences, Vol.7, No.1, 2019, pp. 311-317.

[3] Teng, Ong Yee, Chong Shin Horng, and Hee Wai Keat. "Positioning Control of XY Table Using 2-DOF PID Controller." Applied Mechanics \& Materials 761 (2015).

[4] Hasan, Sami and Aya Moufak Ismael. "Fabrication-Ready Self-Learning PID Controller: A Comparison Study with Classical PID." Journal of Fundamental and Applied Sciences [Online], 10.4S (2018): 654-658. Web. 21 Mar. 2018.

[5] Sevkuthan Kurak and Migdat Hodzic, " Control and Estimation of a Quadcopter Dynamical Model', Periodicals of Engineering and Natural Sciences, Vol.6, No.1, June 2018, pp. 63-75.

[6] Jing Cuil, Zhongyi Chu and Difan Wang, "Iterative learning control of high acceleration positioning table via sensitivity identification', Advances in Mechanical Engineering 2016, Vol. 8(2) $1-11$.

[7] Ma, Letong, et al. "Control of a Magnet-Driven Nano-Positioning Stage with Long Stroke Based on Disturbance Observer." International Conference on Intelligent Robotics and Applications. Springer, Cham, 2017.

[8] Retas, Z., et al. "Tracking error compensation of XY table ball screw driven system using cascade fuzzy P+ PI." Proceedings of Innovative Research and Industrial Dialogue 20161 (2017): 103-104.

[9] Lin, F-J., P-H. Shieh, and P-H. Shen. "Robust recurrent-neural-network sliding-mode control for the XY table of a CNC machine." IEE Proceedings-Control Theory and Applications 153.1 (2006): 111-123.

[10] Giap, Nguyen Hoang, Jin-Ho Shin, and Won-Ho Kim. "Robust adaptive neural network control for XY table." Intelligent Control and Automation 4.03 (2013): 293.

[11] Pietrusewicz, Krzysztof. "Multi-degree of freedom robust control of the CNC XY table PMSMbased feed-drive module." Archives of Electrical Engineering 61.1 (2012): 15-31.

[12] Tahereh Binazadeh, Majid Bahmani, "Robust time-varying output tracking control in the presence of actuator saturation', First Published June 10, 2016, SAGA journals.

[13] Faa-Jeng Lin, Hsin-Jang Shieh, Po-Huang Shieh, Po-Hung Shen, ' Intelligent Motion Control of X-Y Table Using Adaptive Recurrent-Neural-Network', research gate, 2014.

[14] Anupam Kumar, Manoj Kumar Panda, Sumanta Kundu, and Vijay Kumar, ' Designing of an Interval Type-2 Fuzzy Logic Controller for Magnetic Levitation System with Reduced Rule base', 26th_28th July 2012, Coimbatore, India.

[15] Tian Tian, Changyu Liu 1, Qi Guo, Yi Yuan, Wei Li, and Qiurong Yan, ' An Improved Ant Lion Optimization Algorithm and Its Application in Hydraulic Turbine Governing System Parameter Identification', Energies 2018, 11, 95.

[16] Mustafa TINKER, ' A new approach for interval type-2 by using adaptive-network-based fuzzy inference system", International Journal of the Physical Sciences Vol. 6(19), pp. 4502-4518, 16 September 2011.

[17] Swathy Nair, Bindu Elias, VPS Naidu, " Image Fusion Using Type-2 Fuzzy Systems", International Journal of Advanced Research in Electrical, Electronics and Instrumentation Engineering, Vol. 3, Special Issue 5, December 2014.

[18] Djaber Maouche, and Ilyas Eker, 'Adaptive Fuzzy Type-2 in Control of 2-DOF Helicopter', International Journal of Electronics and Electrical Engineering Vol. 5, No. 2, April 2017. 
[19] M. P. Veeraiah S. Majhi Chitralekha Mahanta, "' Fuzzy Proportional Integral - Proportional Derivative (PI-PD) Controller', Proceeding of the 2004 American Control Conference Boston, Massachusetts June 30 - July 2, 2004.

[20] B. Polaj`zer, J. Ritonja, G. `Stumberger, D. Dolinar, J.-P. Lecointe, ' Decentralized PI/PD position control for active magnetic bearings", Electrical Engineering (2005).

[21] Alpesh Patel, Naimesh Mehta, 'Cascade Control System Design and Auto Tuning Using Relay Feedback Technique", INSTITUTE OF TECHNOLOGY, NIRMA UNIVERSITY, AHMEDABAD - 382 481, 09-11 DECEMBER, 2010.

[22] Seyedali Mirjalili, Pradeep Jangir · Shahrzad Saremi, " Multi-objective ant lion optimizer: a multi-objective optimization algorithm for solving engineering problems', Springer, 2016.

[23] Karam, Zeyad A. "Hybrid Fuzzy Congestion Controllers for Computer Networks Sized by Modified Particle Swarm Optimization." International Journal of Advances in Telecommunications, Electrotechnics, Signals and Systems 7.2 (2018): 17-26.

[24] Amjad J. Humaidi, Sami Hassan and Mohammed A. Fadhel, "Rapidly-fabricated nightlydetected lane system: An FPGA-implemented the architecture," The Asian International Journal of Life Sciences 16(1): 343-355, 2018.

[25] Amjad J. Humaidi, Sami Hassan and Mohammed A. Fadhel, "FPGA-based lane-detection architecture for autonomous vehicles: A real-time design and development," The Asian International Journal of Life Sciences 16(1): 223-237, 2018.

[26] Amjad Jaleel Humaidi, Sami Hasan, A Abdulhussein Al-Jodah, "Design of Second Order Sliding Mode for Glucose Regulation Systems with Disturbance," International Journal of Engineering \& Technology, 7 (2.28), 243-247, 2018.

[27] S. Hasan, S. Boussakta and A. Yakovlev, "Improved parameterized efficient FPGA implementations of parallel 1-D filtering algorithms using Xilinx System Generator," The 10th IEEE International Symposium on Signal Processing and Information Technology, Luxor, 2010, pp.

URL: http://ieeexplore.ieee.org/stamp/stamp.jsp?tp=\&arnumber=5711807\&isnumber=5711717

[28] S. Hasan, S. Boussakta and A. Yakovlev, "Parameterized FPGA-based architecture for parallel 1-D filtering algorithms," International Workshop on Systems, Signal Processing, and their Applications, WOSSPA, Tipaza, 2011, pp. 171-174. URL: http://ieeexplore.iee. $.0 r g /$ stamp/stamp.jsp?tp=\&arnumber=5931443\&isnumber=5931402 